\title{
Identification of the minimal region in lipase $A B C$ transporter recognition domain of Pseudomonas fluorescens for secretion and fluorescence of green fluorescent protein
}

Yeonwoo Park ${ }^{1 \dagger}$, Yuseok Moon ${ }^{2+}$, Jungmin Ryoo ${ }^{1}$, Nayeon Kim¹ ${ }^{1}$ Hyounghoon Cho ${ }^{1}$ and Jung Hoon Ahn ${ }^{1 *}$

\begin{abstract}
Background: TliA is a thermostable lipase secreted by the type 1 secretion system (T1SS) of Pseudomonas fluorescens. The secretion is promoted by its secretion/chaperone domain located near the C-terminus, which is composed mainly of four Repeat-in-Toxin (RTX) repeats. In order to identify the minimal region of TliA responsible for its secretion, five different copies of the secretion/chaperone domain, each involving truncated N-terminal residues and a common C-terminus, were acquired and named as lipase ABC transporter recognition domains (LARDs). Each LARD was fused to epidermal growth factor (EGF) or green fluorescent protein (GFP), and the secretion of EGF-LARD or GFP-LARD fusion proteins was assessed in Escherichia coli with ABC transporter.
\end{abstract}

Results: Among the fusion proteins, GFP or EGF with 105-residue LARD3 was most efficiently secreted. In addition, GFP-LARD3 emitted wild type GFP fluorescence. Structurally, LARD3 had the 4 RTX repeats exposed at the $\mathrm{N}$-terminus, while other LARDs had additional residues prior to them or missed some of the RTX repeats. LARD3 was both necessary and sufficient for efficient secretion and maintenance of GFP fluorescence in $E$. coli, which was also confirmed in P. fluorescens and P. fluorescens $\triangle$ tliA, a knock-out mutant of tliA.

Conclusion: LARD3 was a potent secretion signal in T1SS for its fusion flanking RTX motif, which enhanced secretion and preserved the fluorescence of GFP. LARD3-mediated secretion in E. coli or P. fluorescens will enable the development of enhanced protein manufacturing factory and recombinant microbe secreting protein of interest in situ.

Keywords: Green fluorescent protein (GFP), Lipase ABC transporter recognition domain (LARD), ABC transporter, Pseudomonas fluorescens, Secretion/chaperon domain, $\beta$-roll

\section{Background}

The type 1 secretion system (T1SS) secretes polypeptides to the extracellular medium in gram-negative bacteria [1]. T1SS is composed mainly of ATP-binding cassette (ABC) transporter, which is comprised of $\mathrm{ABC}$ protein [2], adaptor or membrane fusion protein (MFP) [3], and outer membrane protein (OMP) [4]. The $\mathrm{ABC}$ protein captures the substrate polypeptide and transports it through the

\footnotetext{
* Correspondence: hoony@kaist.ac.kr

${ }^{\dagger}$ Equal contributors

'Korea Science Academy of KAIST, 899 Tanggam 3-Dong, Busanjin-Gu, Busan 614-822, Korea

Full list of author information is available at the end of the article
}

contiguous channel formed by MFP and OMP by coupling ATP hydrolysis [5-7]. The MFP-OMP channel penetrates both gram-negative membranes simultaneously, leaving no periplasmic intermediates [8]. T1SS is also referred to as a signal peptide-independent secretion system because the secretion is mediated by the C-terminal region of a substrate polypeptide instead of the signal sequence [9].

TliA is a 476 aa thermostable lipase encoded in the lipase operon of Pseudomonas fluorescens. TliA is secreted by a dedicated T1SS composed of TliDEF, an ABC transporter encoded in the upstream of TliA within the lipase operon [10]. TliA is exported by TliDEF in high efficiency at $25^{\circ} \mathrm{C}$, which is the optimum growth temperature of $P$. fluorescens

\section{C) Biomed Central}

(c) 2012 Park et al.; licensee BioMed Central Ltd. This is an Open Access article distributed under the terms of the Creative Commons Attribution License (http://creativecommons.org/licenses/by/2.0), which permits unrestricted use, distribution, and reproduction in any medium, provided the original work is properly cited. 
[10]. By homology, TliA is also exported by PrtDEF, the $\mathrm{ABC}$ transporter of Erwinia chrysanthemi, at $37^{\circ} \mathrm{C}$ [11]. TliA has a lipase activity domain, a hinge region, a secretion/chaperone domain, and a calcium-binding domain (4 RTX repeats) in residues 1-268, 269-278, 279-476, and 373-417, respectively [11].

Several T1SS substrate polypeptides have been widely studied. Those include HlyA from Escherichia coli [12,13], PrtB and PrtC from E. chrysanthemi [14,15], AprA $_{\mathrm{PA}}$ from Pseudomonas aeruginosa [16], and TliA from P. fluorescens [10]. These substrate polypeptides share common structures such as an extreme Cterminal motif [17], a hydrophobic five-residue sequence motif (VTLIG) [18,19] and a Repeat-in-Toxin (RTX) motif, GGxGxDxUx repeats (x: any amino acids; U: large hydrophobic residues such as $\mathrm{L}$, I, or F). According to the crystal structures of $\operatorname{AprA}_{\mathrm{PA}}[20,21]$ and $\operatorname{PrtA}_{\mathrm{SM}}$ $[22,23]$, the RTX motif forms a $\beta$-roll structure in which two RTX repeats constitute a complete turn involving a short parallel $\beta$-sheet. The RTX motif has also been referred to as a calcium-binding domain [9,16,24,25], because $\mathrm{Ca}^{2+}$ ions are required for the stabilization of the $\beta$-roll structure [26]. The exact role of the RTX motif has not been pinpointed, but it is known to affect receptor binding [27], enhance secretion [28], act as an internal chaperone [21], and increase protein levels in the cytoplasm [29]. T1SS substrate polypeptides have no strictly-defined residues required for secretion, but the overall structure including the RTX motif determines the secretion.

In this research, the minimal region in TliA responsible for its secretion was investigated. Five different copies of the secretion/chaperone domain, the C-terminal region of TliA involving the RTX motif, were obtained. These different lengths of polypeptides, defined as the lipase $A B C$ transporter recognition domain (LARD), were attached to the C-terminus of epidermal growth factor (EGF) or green fluorescent protein (GFP). The fusion proteins were expressed with appropriate T1SS in E. coli, P. fluorescens, and $P$. fluorescens $\Delta$ tliA, a knock-out mutant lacking tliA in its genome. As a result, LARD3 was necessary and sufficient for efficient secretion. In addition, several observations suggested the RTX motif as both a secretion enhancer and an internal chaperone.

\section{Results}

Construction of GFP-LARD and EGF-LARD fusion proteins

LARD1 to 5 were constructed by PCR amplifying the secretion/chaperone domain of TliA from residues 303, 337, 373, 407, and 442 to the C-terminus (residue 476), respectively. The features of the secretion/chaperone domain and LARDs are shown (Figure 1A). LARDs were fused to the C-terminus of GFP to construct GFP-LARD fusion proteins with a Factor Xa protease cleavage site as a linker. EGF-fusion proteins were constructed in a similar manner as GFP-fusion proteins. The threedimensional structures of LARDs predicted by SWISSMODEL structural modeling program [30] are also represented (Figure 1B). LARD1 (174 aa) included 70 additional residues prior to the 4 RTX repeats. Those residues were partly removed in LARD2 (140 $a a)$ and completely removed in LARD3 (104aa). As a result, the RTX motif was exposed at the N-terminus of LARD3. Three out of 4 RTX repeats were removed in LARD4 (70 aa), and LARD5 (35 aa) was deprived of any common structures except for the extreme C-terminal motif.

\section{Expression and secretion of EGF-LARDs in E. coli}

The expression and secretion of EGF-LARD1 to 5 and EGF-TliA were examined in E. coli XL1-Blue with or without PrtDEF, the ABC transporter of E. chrysanthemi. The expression of EGF-fusion proteins, as analyzed by western blotting, was heterogeneous (Figure 2). EGFLARD3 was undetected in the cell when it was coexpressed with PrtDEF. However, it was detected inside the cell when PrtDEF was absent. The same result was also observed in E. coli MM294. Because it is close to the original strain E. coli K12 [31], exhibiting wild-type phenotype, and showed strong fluorescence when transformed with GFP gene in our experiment, E. coli MM294 was used together with XL1-Blue throughout the experiments. However, we do not draw any conclusion by contrasting the results obtained from the two strains as our purpose in this research is to draw a generalization that applies to a wide range of species.

For extracellular secretion, EGF-LARD3, EGF-LARD4 and EGF-TliA were secreted from E. coli XL1-Blue (Figure 3A) while only EGF-LARD3 was secreted from E. coli MM294 (Figure 3B). Since EGF-LARD3 was detected only in the extracellular medium when the cells co-expressed PrtDEF, and was detected solely in the cytoplasm when the cells did not express PrtDEF, it was evident that secretion occurred through the ABC transporter instead of other possible pathways such as cell rupture. The concentration of the secreted EGF-LARD3 was about $3.3 \mu \mathrm{g} / \mathrm{ml}$ as determined by comparing to the purified lipase solution.

\section{Expression and secretion of GFP-LARDs in E. coli}

The intracellular expression of GFP-LARDs in E. coli was analyzed by western blotting (Figure 4). GFPLARDs and GFP-TliA were uniformly expressed by $E$. coli cells harboring pGFP-LARD1 to 5 or pGFP-TliA, each with $\mathrm{pEcPrtDEF}$ (the vector harboring prtDEF). The secretion pattern of different GFP-LARD fusion proteins was analyzed in E. coli XL1-Blue (Figure 5A) and E. coli MM294 (Figure 5B). In contrast to EGF, GFP was secreted by many types of LARDs except for 


\section{A}

301-

†303, LARD1

PFSIANLSTWVSHLPSAYGDGMTRVLESGFYEQMTRDSTIIVA

NLSDPARANTWVQDLNRNAEPHTGNTFII GSDGNDLIQGGKG

ADFIEGGKGNDTIRDNSGHNTFLFSGHFGQDRIIGYQPTDRLVF

QGADGSTDLRDHAKAVGADTVLSFGADSVTLVGVGLGGLWS

EGVLIS -476

Repeat-in-Toxin motifs (GGXGXDXUX repeats)

Hydrophotic five-sequence motif

Extreme C-terminal motif

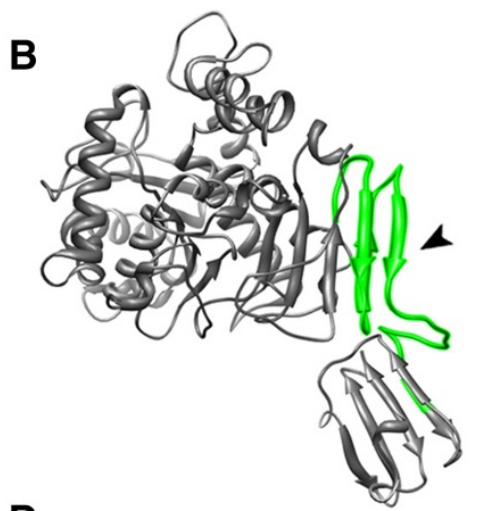

D

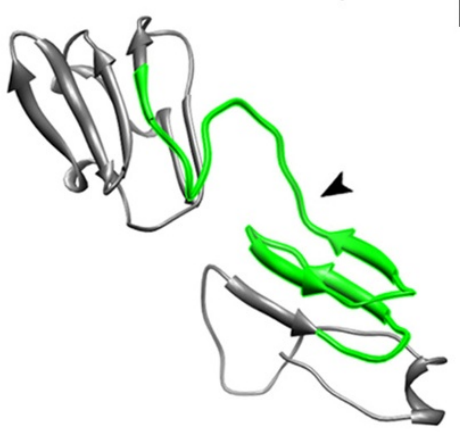

$\mathbf{F}$

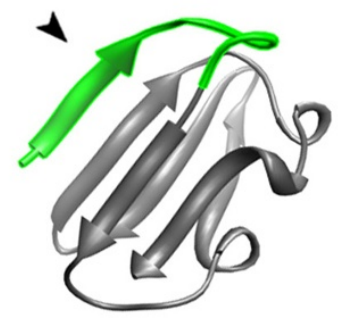

C

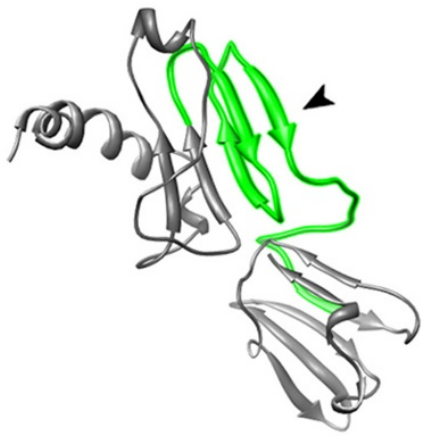

E

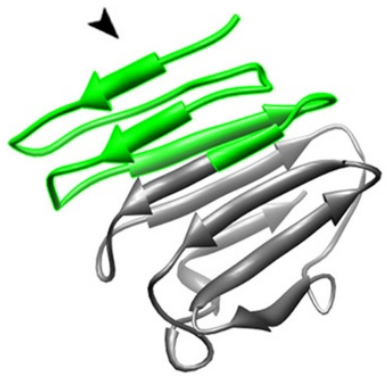

G

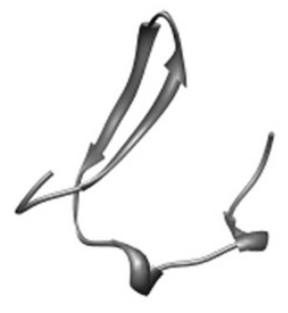

Figure 1 The features and structures of LARDs. A. The secretion/chaperone domain of TliA (residues 301-476). The residues and PCR amplification sites of LARDs are also shown overlapped. TliA has a calcium-binding domain (373-416) comprised of four GGxGxDxUx repeats, hydrophobic five-sequence motif and an extreme C-terminal motif, EGVLIS. The three-dimensional structures of each LARDs were predicted by SWISS-MODEL structural modeling according to their residues and displayed by UCSF Chimera (http://www.cgl.ucsf.edu/chimera/) [30]. The RTX motif is indicated by the arrowhead. B. TliA, C-G. LARD1 to 5. Note that LARD3 has the RTX motif exposed at the N-terminus. The protein data bank (PDB) IDs for the templates used to predict each structure are as follows: TliA, 2z8z_A; LARD1, 2z8z_A; LARD2, 2qub_A; LARD3, 2zvd_C; LARD4, 2qub_A; LARD5; 2zvd_C. 


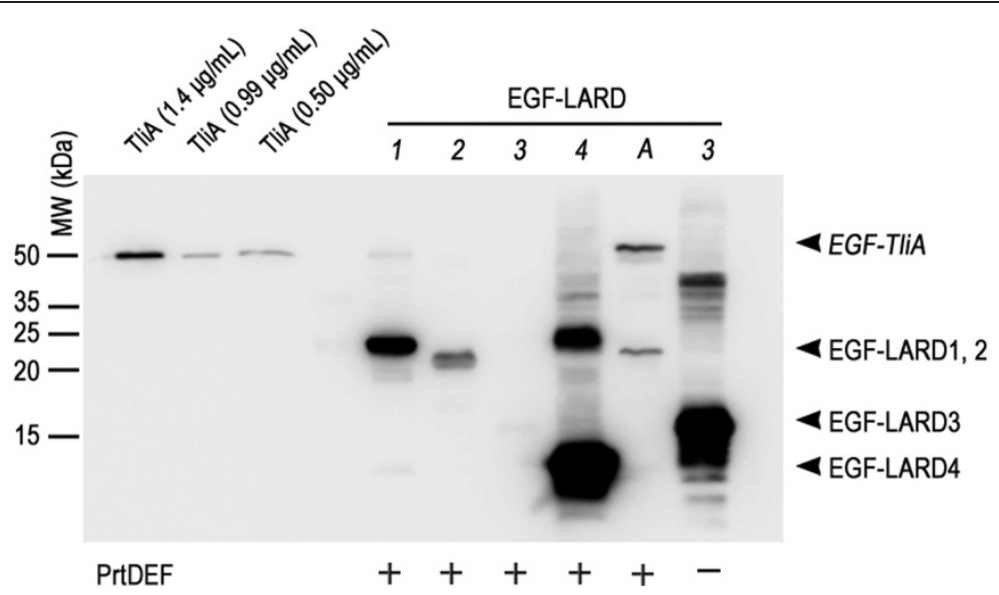

Figure 2 The intracellular expression of EGF-LARDs in E. coli XL1-Blue. Each E. coli XL1-Blue culture harboring one of pEGF-LARD1-4 (lanes 1-4) or pEGF-TliA (lane A) with pEcPrtDEF (+) or pACYC-184 (-) was incubated with $0.05 \mathrm{mM} \mathrm{IPTG,} 50 \mu \mathrm{g} / \mathrm{ml}$ ampicillin, and $34 \mu \mathrm{g} / \mathrm{ml}$ chloramphenicol in $2 \mathrm{ml}$ glass tubes at $37^{\circ} \mathrm{C}$. The cells were isolated from the extracellular medium by double centrifugation, underwent SDS-PAGE in 15\% gel, and analyzed by western blotting using anti-LARD antibodies. The plasmid PACYC-184 was used as a negative control for pECPrtDEF which encodes PrtDEF. Purified TliA at a concentration of $99 \mu \mathrm{g} / \mathrm{ml}$ was used as an index after dilution. The arrowheads indicate the size of each EGF-LARD.

LARD5. Among the fusion proteins, GFP-LARD3 showed the greatest concentration in the extracellular medium. This pattern was more evident in E. coli MM294 than in XL1-Blue, the concentration of GFPLARD3 in the former being approximately $10 \mu \mathrm{g} / \mathrm{ml}$.

The GFP fluorescence of different GFP-LARD fusion proteins was analyzed under UV light (Figure 6A) and quantitatively measured by fluorescence spectroscopy (Figure $6 \mathrm{~B}$ ). At $25^{\circ} \mathrm{C}$ which is the optimum growth temperature of $P$. fluorescens, the host species of TliA from which LARDs are derived, GFP-LARD3 showed the highest fluorescence in both $E$. coli XL1-Blue and
MM294, exhibiting 96\% that of the wild type GFP in MM294. As the temperature increased from $25^{\circ} \mathrm{C}$ to $37^{\circ} \mathrm{C}$, the fluorescence decreased for GFP-LARD3. Other GFPLARD fusion proteins showed a similar decrease in fluorescence in E. coli XL1-Blueand MM294.

Further analyses on the expression and secretion of GFP-LARDs in $P$. fluorescens were undertaken with GFP-LARD3 and GFP-TliA which were suitable for detection in the western blotting and lipase activity plate, respectively. The GFP-TliA was detected easily on the tributyrin plate when it was secreted by $\mathrm{ABC}$ transporter, showing halos around colonies.
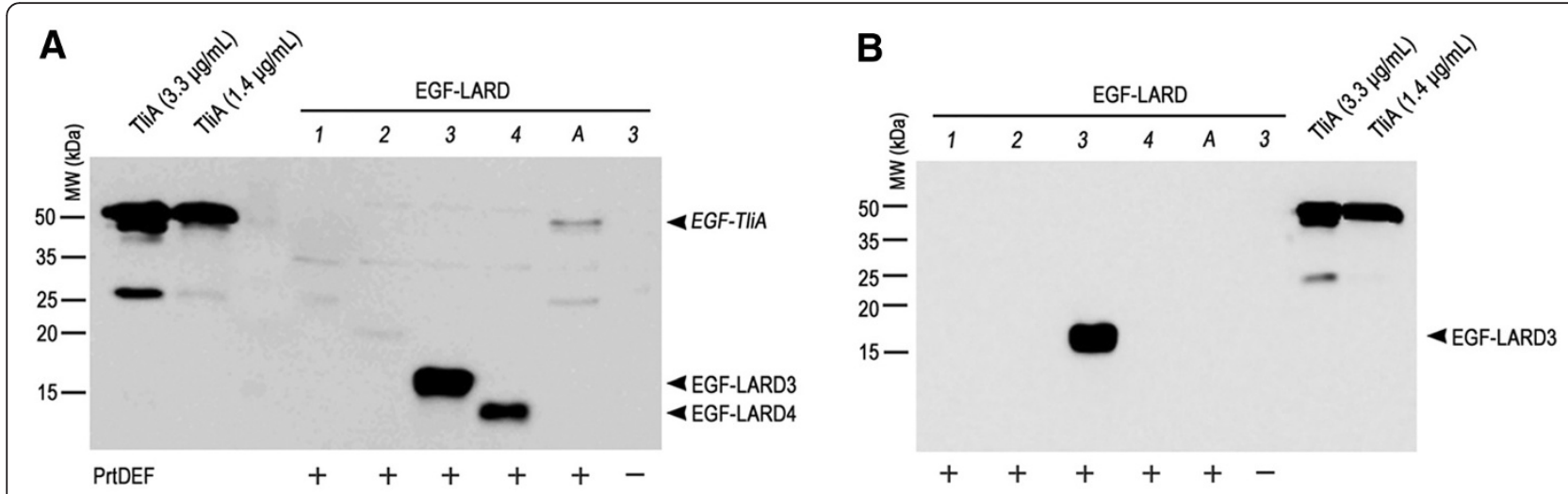

Figure 3 The extracellular secretion of EGF-LARDs in E. coli XL1-Blue and MM294. A. The secretion of EGF-LARDs in E. coli XL1-Blue. Each culture harboring one of pEGF-LARD1-4 (lanes 1-4) or pEGF-TliA (lane A) with pEcPrtDEF was centrifuged twice, and the supernatant was collected. The growth conditions were identical to those in Figure 2. SDS-PAGE with 15\% gel and western blotting with anti-LARD antibodies were performed on the supernatant. The $(+)$ sign indicates the presence of PrtDEF. The $(-)$ sign indicates the absence of PrtDEF (pACYC-184 was used as a negative control). Purified TliA at $99 \mu \mathrm{g} / \mathrm{ml}$ concentration was used as an index after dilution. B. The secretion of EGF-LARDs in E. coli MM294. The methods used here were identical to those used in (A). 


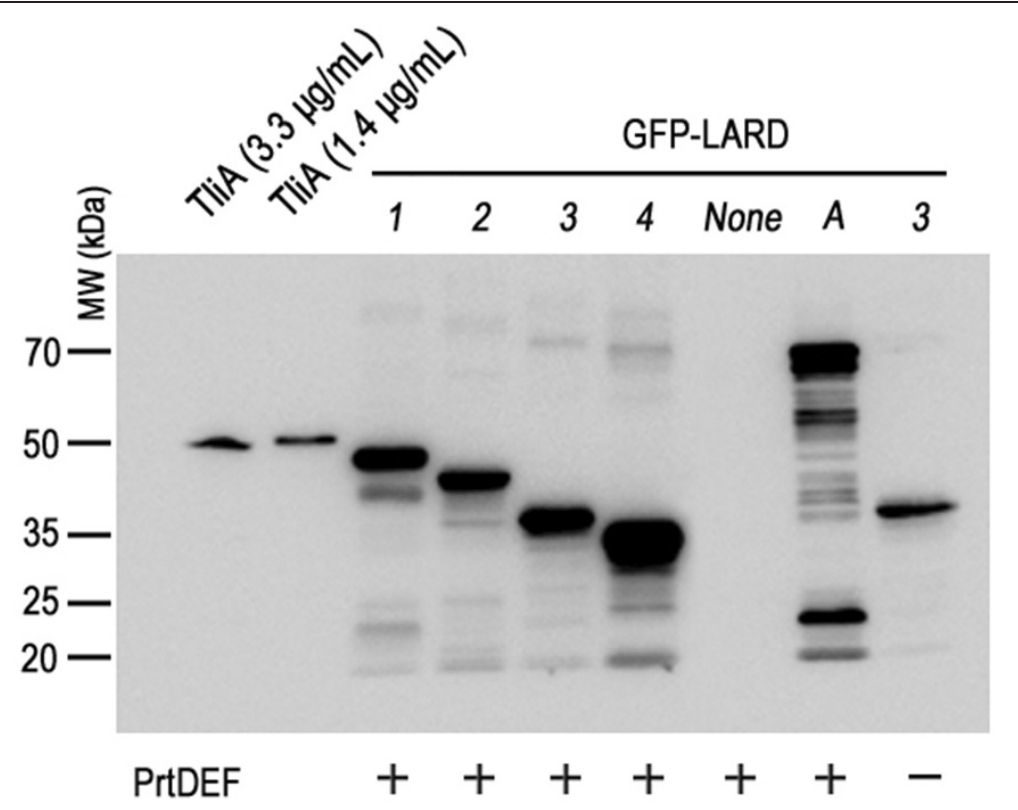

Figure 4 The intracellular expression of GFP-LARDs in E. coli XL1-Blue. Each E. coli XL1-Blue culture harboring one of PGFP-LARD1-4 (lanes 1-4), pGFP-TliA (lane A) or pGFP-223 (lane None) with pECPrtDEF (+) or pACYC-184 (-) was grown with $50 \mu \mathrm{g} / \mathrm{ml}$ ampicillin and $34 \mu \mathrm{g} / \mathrm{ml}$ chloramphenicol in a $2 \mathrm{ml}$ glass tube at $37^{\circ} \mathrm{C}$ (IPTG was not added). The culture was centrifuged twice, and the pellet was isolated. SDS-PAGE with $10 \%$ gel and western blotting with anti-LARD antibodies were performed on the pellet. Purified TliA at a concentration of $99 \mu \mathrm{g} / \mathrm{ml}$ was used as an index after dilution.

\section{Expression and secretion of GFP-LARD3 and GFP-TliA in $P$.} fluorescens

LARDs were derived from a lipase of P. fluorescens. To check whether GFP-LARD fusion proteins were secreted better in the original host, the fusion proteins and an $A B C$ transporter were introduced into $P$. fluorescens. In the former experiments, the GFP-fusion proteins were secreted in E. coli through a two-vector system in which the coding sequences of GFP-fusion proteins were inserted in pKK223 and that of the ABC transporter, PrtDEF, was inserted in pACYC184. The plasmids used for $E$. coli could not be used in P. fluorescens, so a broad
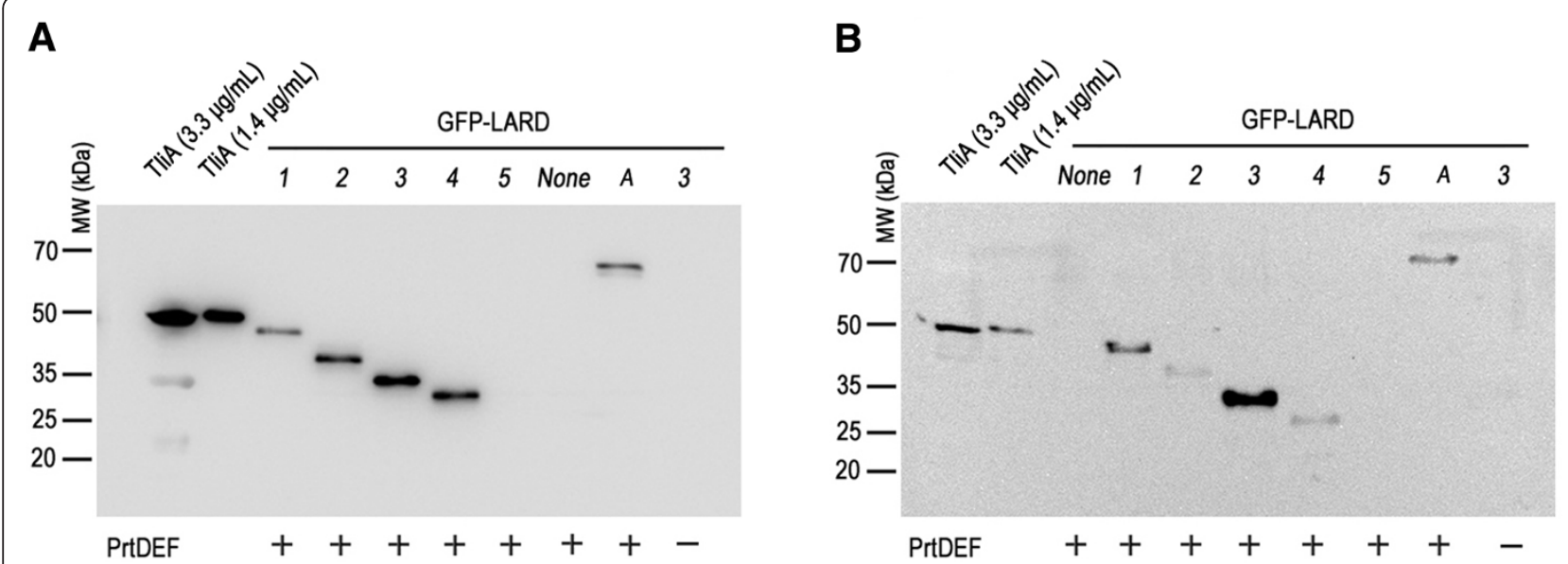

Figure 5 The extracellular secretion of GFP-LARDs in E. coli XL1-Blue and MM294. A. The secretion of GFP-LARDs in E. coli XL1-Blue. Each E. coli XL1-Blue culture harboring one of pGFP-LARD1-5 (lanes 1-5), pGFP-TliA (lane A) or pGFP-223 (lane None) with pECPrtDEF (+) or pACYC-184 $(-)$ was centrifuged twice, and the supernatant was collected. The growth conditions were identical to those listed in Figure 4. SDS-PAGE with $10 \% \mathrm{gel}$ and western blotting with anti-LARD antibodies were performed on the supernatant. Purified TliA at a concentration of $99 \mu \mathrm{g} / \mathrm{ml}$ was used as an index after dilution. B. The secretion of GFP-LARDs in E. coli MM294. The methods used here were identical to those listed in A. The signs are also identical to those of $\mathrm{A}$. 


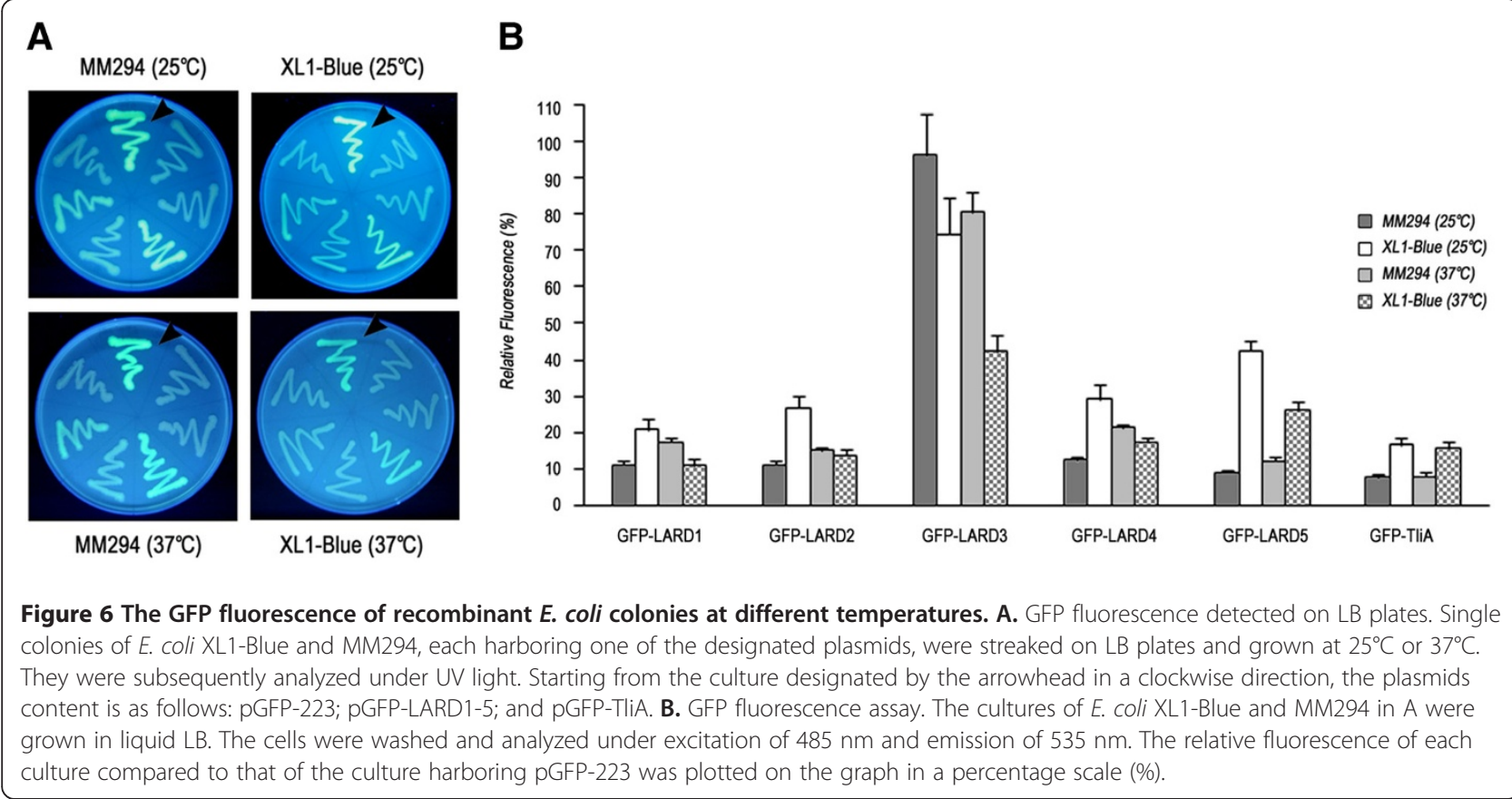

host range vector was constructed; tliDEF, the ABC transporter encoded in the lipase operon of $P$. fluorescens, was inserted into pDSK-519 together with the coding sequences of GFP-LARD3 and GFP-TliA. A similar result obtained from E. coli (Figure 6) was also observed when $P$. fluorescens cells harboring above plasmids were assayed for GFP fluorescence (Figure 7). As expected, in $P$. fluorescens, GFP-LARD3 had a comparable GFP fluorescence to that of an intact GFP. P. fluorescens harboring pDX-GFP-TliA showed a low fluorescence comparable to that of wild type P. fluorescens.

The intracellular and extracellular expressions of GFPLARD3 and GFP-TliA in P. fluorescens were analyzed by western blotting using anti-LARD antibodies (Figure 8). The P. fluorescens cells harboring pDSK-TliA, pDX-TliA, pDX-GFP-LARD3, and pDX-GFP-TliA were grown in LB or $2 \times$ LB media. The intracellular expression was uniform for each protein in each growth condition. The intrinsic TliA (encoded by the genomic tliA) was detected in every extracellular medium, as indicated by the arrow. Here, TliA was also secreted by the intrinsic $\mathrm{ABC}$ transporter in the lipase operon of P. fluorescens (Figure 8, lane 1). GFP-LARD3 was more efficiently exported than GFP-TliA in P. fluorescens, as was the case in $E$. coli. The secretion efficiency was generally higher when the fusion proteins were exported by TliDEF in P. fluorescens than by PrtDEF in E. coli. The intrinsic TliA was secreted more favorably by TliDEF in that some fractions of GFP-LARD3 and GFP-TliA were left inside the cells while all intrinsic TliA was found outside the cells (Figure 8, lane 3 and 4). Further analyses were undertaken with $P$. fluorescens $\Delta t l i A$ to eliminate the intrinsic TliA from the extracellular medium.

\section{Expression and secretion of GFP-LARD3 and GFP-TliA in $P$. fluorescens $\Delta$ tliA}

P. fluorescens $\triangle t l i A$, the genomic tliA-deficient strain, was used to detect the intracellular expression and extracellular secretion of GFP-LARD3 and GFP-TliA (Figure 9). Cells harboring pDSK-TliA, pDX-TliA, pDXGFP-LARD3, and pDX-GFP-TliA were analyzed by western blotting. The patterns were identical to those observed in the wild type $P$. fluorescens, except that the intrinsic TliA was absent in the extracellular medium of pDX-GFP-LARD3. GFP-LARD3 was detected inside the cell and in the extracellular medium without TliA. However, although the genomic tliA was deleted, both TliA and GFP-TliA were found in the extracellular medium of the cells that expressed only GFP-TliA. When the same intracellular and extracellular media were analyzed using anti-GFP antibodies, GFP was found as its monomeric size in the extracellular medium while only the intact GFP-TliA was detected inside the cell (data not shown). It appears that GFP-TliA was degraded by proteolysis after secretion. Experiments with $P$. fluorescens $\triangle p r t A$, which was constructed recently in our laboratory, showed that GFP-TliA was indeed degraded by the protease PrtA which is encoded in the lipase operon and is also secreted by TliDEF [10]. The degradation of extracellular GFP-TliA was not observed in E. coli as shown in previous figures. 


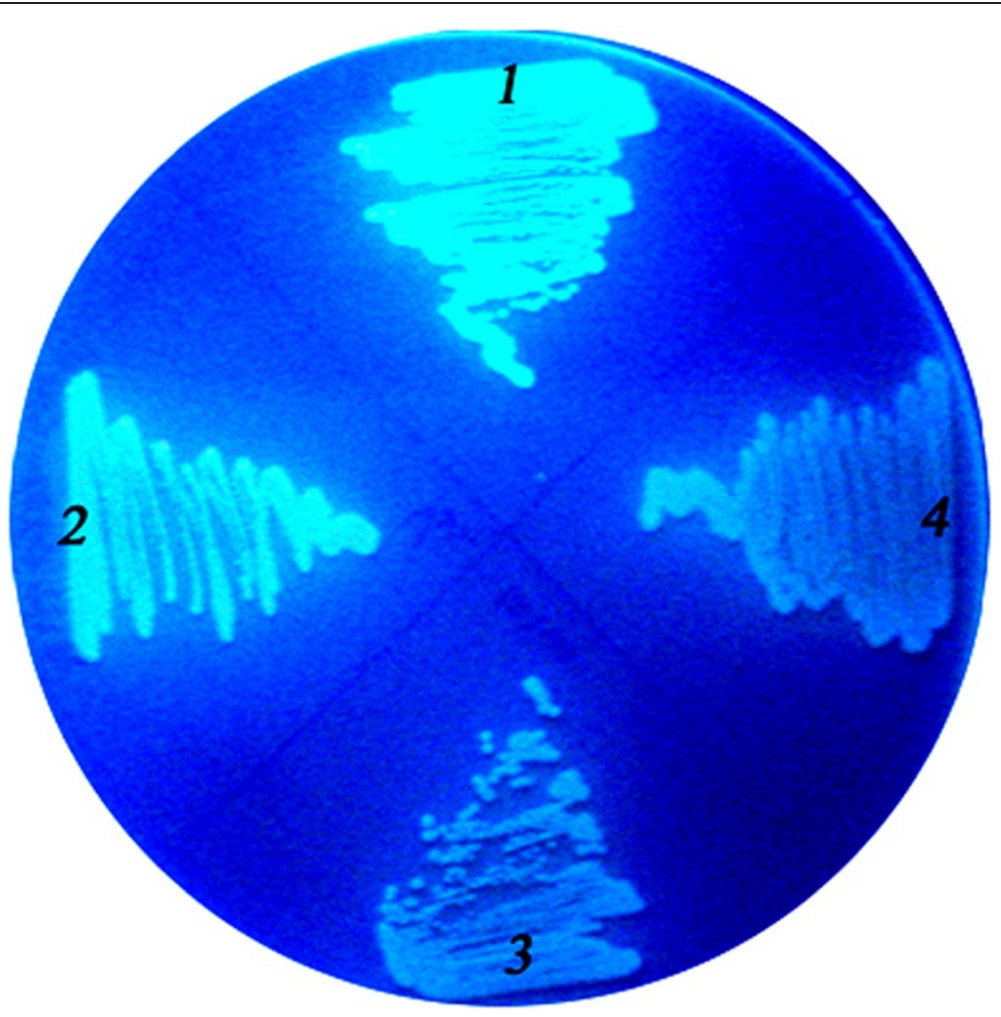

Figure 7 The GFP fluorescence of recombinant $P$. fluorescens colonies. The recombinant $P$. fluorescens cells harboring appropriate plasmids were cultured on an LB plate, and the GFP fluorescence was analyzed using UV light. Label 1, pDSK-TliDEF-GFP-LARD3 (pDX-GFP-LARD3); label 2, pDX-GFP-223; label 3, pDX-TliA; label 4, pDX-GFP-TliA.

\section{Discussion}

In this research, LARD has been reported to be a secretion signal capable of exporting GFP or EGF through the gram-negative T1SS. LARD1 to 5 were acquired from the secretion/chaperone domain of TliA, and they involved part of the homologous structures essential for secretion, such as the RTX repeats, hydrophobic fiveresidue sequence motif and an extreme $\mathrm{C}$-terminal motif. LARDs were attached to GFP or EGF, and the fusion proteins were expressed in E. coli and P. fluorescens with PrtDEF or TliDEF. The secretion was observed with different LARD fusion proteins, among which EGFLARD3 and GFP-LARD3were most efficient in the secretion of EGF and GFP. In addition, GFP-LARD3 retained its native GFP fluorescence, while the other fusion proteins had a diminished fluorescence.

The GFP fluorescence was preserved particularly in GFP-LARD3. Note that LARD3 had the 4 RTX repeats exposed at the $\mathrm{N}$-terminus, while others had additional residues prior to them or missed several repeats. It appeared that in GFP-LARD3, the RTX repeats had preserved GFP by structurally separating it from the remaining residues of LARD3. It is yet uncertain whether or not RTX repeats form a stable $\beta$-roll structure or remain intrinsically disordered in the cytosol, but in either case, they were able to preserve GFP by acting like a structural barrier. In support of this hypothesis, Blenner et al. provided evidence of the RTX motif as a distinct domain that can be folded or unfolded independently to the rest of the polypeptide [32]. Either as a $\beta$-roll or as an intrinsically disordered structure, the RTX repeats were able to separate GFP from the secretion signal.

The exact secretion mechanism of T1SS is still being researched. Relevant findings in understanding the mechanism of T1SS include: 1) that the substrate polypeptides sequentially form a multi-protein complex with the $\mathrm{ABC}$ protein, MFP, and OMP prior to secretion [33]; and 2) RTX repeats are intrinsically disordered in the calcium-free cytosol and keep the substrate polypeptide translocation-competent [32,34]. Once exported to the calcium-rich extracellular environment, RTX repeats structuralize under conditions involving entropic stabilization and become an internal chaperone. This spontaneous calcium-induced folding in the extracellular medium prevents the backtracking of the substrate polypeptide and enhances export by conferring directionality towards the outside of the bacterium, a process known 

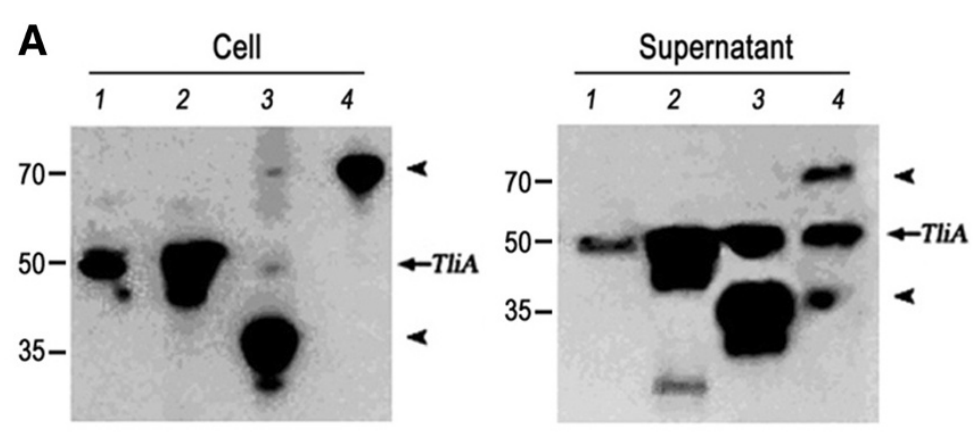

B
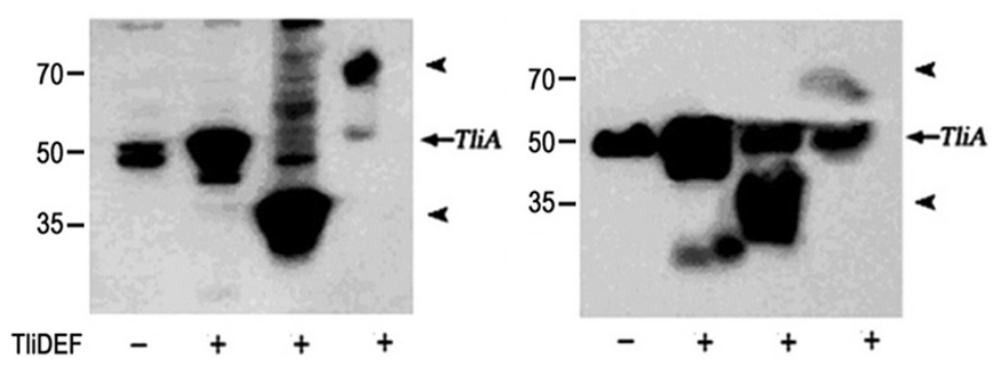

Figure 8 The expression and secretion of the recombinant proteins in P. fluorescens. A. The intracellular expression and secretion of GFP-TliA and GFP-LARD3 in P. fluorescens cultured in LB were detected by western blotting using anti-LARD antibodies. The $P$. fluorescens cells expressing the designated proteins with (+) or without (-) TliDEF were isolated from two different extracellular media by double centrifugation. B. The same expression was performed in 2x LB (double concentrated LB). Lane 1, pDSK-TliA; lane 2, pDX-TliA; lane 3, pDX-GFP-LARD3; lane 4, pDX-GFP-TliA. The arrows indicate the sizes of GFP-LARD3 (38.2 kDa), TliA (49.9 kDa), and GFP-TliA (77.4 kDa). Notice the intrinsic TliA indicated by the arrows.

as the ratchet mechanism [34]. Those findings indicate that there are complex intermediate protein-protein interactions involved in secretion. The fundamental source of these complications is the C-terminal localization of the secretion signal; a complete translation is required prior to secretion. Since the export passage is too narrow for a folded globular protein to pass, there must be some mechanism that retains the synthesized substrate polypeptide relatively unfolded and translocation-competent [35].

There is a paradox, in that GFP-LARD3 had a native fluorescence while being most efficiently secreted. The

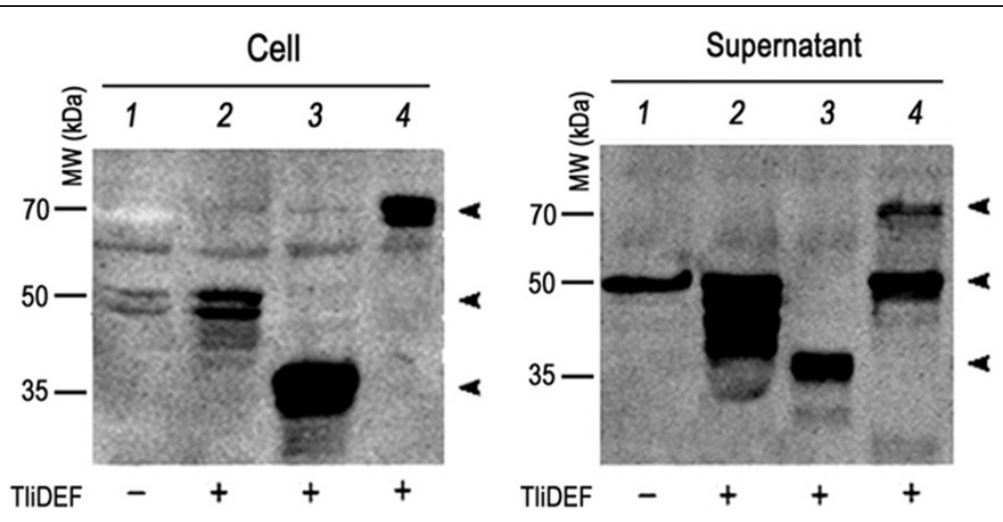

Figure 9 The expression and secretion of the recombinant proteins in $P$. fluorescens $\Delta t$ tiA. The intracellular expression and secretion of GFP-TliA and GFP-LARD3 were analyzed by western blotting using anti-LARD antibodies. The cells were isolated from the growth medium by double centrifugation. Lane 1, pDSK-TliA; lane 2, pDX-TliA; lane 3, pDX-GFP-LARD3; lane 4, pDX-GFP-TliA. The arrows indicate the sizes of GFPLARD3 (38.2 kDa), TliA (49.9 kDa), and GFP-TliA (77.4 kDa). 
preserved fluorescence implies a stably-folded GFP, while the high secretion efficiency indicates the intrinsicallydisordered, relatively unfolded, translocation-competent state of the fusion protein [36]. The more compactly the GFP was folded, the harder it become for the fusion protein to pass through the MFP-OMP channel, decreasing the secretion efficiency. This seeming contradiction implied a more complex state of the substrate polypeptide during secretion, such as being locally folded at the fused domain (GFP in this case) and unfolded at the secretion signals. Also, upon binding extracellular $\mathrm{Ca}^{+2}$ ions, the secreted GFP-LARD fusion proteins would probably assume folded C-terminal regions, but the folding of the GFP in the extracellular medium is uncertain because only low fluorescence was detected in the extracellular medium. In the case of EGF, EGF-LARD3 shows normal EGF function after secretion [37]. The structure and function of secreted proteins were not sure but the proteins were assumed to be folded after the secretion but possibly failed to fold into normal structure.

\section{Conclusions}

The RTX motif directly linked with fusion protein enhanced secretion and preserved the fluorescence of GFP. The 4 RTX repeats in TliA were not strictly required for secretion as LARD4, which is deficient of 2 out of 4 RTX repeats, was still secreted. However, as LARD3 showed, the secretion was most efficient in the presence of all 4 RTX repeats without any additional $\mathrm{N}$-terminal residues. LARD3 was a minimal region in TliA capable of exporting GFP and maintaining its fluorescence. LARD3 was recently used to make a transgenic probiotic microbe secreting EGF for enhanced would healing [37]. LARD3 presents a suitable tool to produce useful recombinant proteins extracellularly in $E$. coli or in P. fluorescens, and even more, to design an in situ protein manufacturing factory utilizing living microbes in producing and secreting proteins of interest.

\section{Methods}

\section{Bacterial strains and plasmids}

E. coli XL1-Blue, E. coli MM294, and P. fluorescens SIK W1 (KCTC 7689) were used as hosts for DNA manipulation and gene expression. E. coli MM294 ( $\mathrm{F}^{-}$endA1 hsdR17 $\left(\mathrm{r}_{\mathrm{K}}^{-} \mathrm{m}_{\mathrm{K}}^{+}\right) \operatorname{gln} V 44$ thi-1 relA1 rfbD1 spoT1) was used as expression host because GFP was expressed well to show strong fluorescence. This strain is very close to wild type E. coli K-12 [31]. P. fluorescens SIK W1 $\Delta$ tliA, a knock-out mutant lacking the genomic TliA, was constructed in the authors' laboratory. Plasmids pKK223-3, pDSK-519, and pACYC-184 were used as expression vectors. The plasmids were introduced into $E$. coli cells by heat transformation and into P. fluorescens cells by electroporation or conjugation using E. coli F-positive strain S17-1 as a donor.

\section{Expression of fusion proteins}

E. coli was grown in Luria-Bertani (LB) at $37^{\circ} \mathrm{C}$ for one day in an orbital shaker at $150 \mathrm{rpm}$. For the expression of fusion proteins, $E$. coli was grown in $2 \mathrm{ml}$ medium in a $15 \mathrm{ml}$ culture tube. Isopropyl $\beta$-D-1-thiogalactopyranoside (IPTG) was added at $0.05 \mathrm{mM}$ for the expression of pKK223-3-derived plasmids which is under tac promoter. For dual plasmid expression, $50 \mu \mathrm{g} / \mathrm{ml}$ ampicillin was used to maintain the plasmids derived from $\mathrm{pKK} 223-3$, and $34 \mu \mathrm{g} / \mathrm{ml}$ chloramphenicol was used to maintain the pACYC-184 derivatives. P. fluorescens was grown in $\mathrm{LB}$ or $2 \times \mathrm{LB}$ media at $25^{\circ} \mathrm{C}$ for 2 days. The $2 \times$ LB medium is a doubly concentrated LB medium used for specific growth of $P$. fluorescens. P. fluorescens was grown in $8 \mathrm{ml}$ medium in a $20 \mathrm{ml}$ glass tube at $150 \mathrm{rpm}$ to reduce the degradation of proteins by aeration [38]. pDSK519-derived plasmids were under lac promoter but IPTG was not used because expression of genes was constitutive in P. fluorescens [38]. For one vector system in P. fluorescens, $30 \mu \mathrm{g} / \mathrm{ml}$ kanamycin was used to maintain the pDSK-519 derivatives.

\section{Plasmid construction}

The plasmids used in this research are summarized in Table 1. The GFP gene from pGFPuv (Clontech, Mountain View, CA) and LARD coding sequences from pTOTAL were PCR amplified using primers with EcoRI/XbaI and XbaI/HindIII sites, respectively. Factor Xa protease cleavage site (IEGR) was added between the GFP and LARDs as a linker by attaching the corresponding oligonucleotide to the primers. The GFP and LARD sequences were inserted respectively into EcoRI-XbaI and XbaI-HindIII sites of pKK223-3 downstream of the tac promoter to construct pGFP-LARD1 to 5. The EGF gene was PCR-amplified using EGF-containing plasmid pGEM-hEGF and EGFfusion proteins were constructed in a similar manner as GFP-fusion proteins. The prtDEF gene from Erwinia chrysanthemi was inserted into SacI-NdeI site of pACYC184 to construct pEcPrtDEF. Those plasmids were introduced simultaneously into E. coli XL1-Blue or MM294 via heat transformation. The tliA and tliDEF genes were inserted into pDSK-519 under the lac promoter to construct pDSK-TliA and pDSK-TliDEF (abbreviated as pDX throughout this paper), respectively. In addition, the coding sequences for GFP, TliA, GFP-LARD3, and GFP-TliA were inserted into KpnI-SacI site of pDX downstream of the tliDEF to construct pDX-GFP-223, pDX-TliA, pDX-GFPLARD3, and pDX-GFP-TliA, respectively. Those plasmids were introduced into E. coli S17-1 cells by heat transformation prior to conjugation.

\section{LARD design}

TliA has a lipase activity domain in residues 1-268, a hinge region in residues 269-278, a secretion/ 
Table 1 The plasmids used in this research

\begin{tabular}{|c|c|c|}
\hline Plasmids & Characteristics & Reference \\
\hline pKK223-3 & Cloning vector, $A p^{r}$ & Amersham \\
\hline pACYC-184 & Cloning vector, $\mathrm{Cm}^{\mathrm{r}}$ & $\begin{array}{l}\text { New England } \\
\text { Biolabs }\end{array}$ \\
\hline pDSK-519 & Cloning vector, $\mathrm{Km}^{\mathrm{r}}$ & {$[39]$} \\
\hline pGFPuv & gfp & Clontech \\
\hline pTOTAL & P. fluorescens tliA operon & {$[10]$} \\
\hline pGFP-TliA & gfp-tliA in pKK223-3 & This study \\
\hline pGFP-LARD1 to 5 & gfp-lard1 to 5 in pKK223-3 & This study \\
\hline pEGF-LARD1 to 5 & egf-lard1 to 5 in pKK223-3 & \\
\hline pEcPrtDEF & prtDEF in pACYC-184 & This study \\
\hline pDSK-TliA & tliA in pDSK-519 & This study \\
\hline pDSK-TliDEF (pDX) & tliDEF in pDSK-519 & This study \\
\hline pDX-GFP-223 & gfp and tliDEF in pDSK-519 & This study \\
\hline pDX-TliA & tliA and tliDEF in pDSK-519 & This study \\
\hline pDX-GFP-TliA & gfp-tliA and tliDEF in pDSK-519 & This study \\
\hline pDX-GFP-LARD3 & gfp-lard3 and tliDEF in pDSK-519 & This study \\
\hline
\end{tabular}

chaperone domain in residues 279-476, and a calcium-binding domain in residues 373-417 [11]. The location of PCR amplification sites for the design of LARDs was determined according to the three-dimensional structure of TliA predicted by the SWISS-MODEL structural modeling program at http://swissmodel.expasy.org/ [30]. Appropriate PCR primers were constructed to acquire five different LARDs each elongating from residues 303, 337, 373, 407, and 442 to the C-terminus. The oligonucleotides for the Factor $\mathrm{Xa}$ protease cleavage site were added to the primers, and the primers were used to fuse the LARDs to the C-terminus of GFP by means of ligation to construct GFP-LARD fusion proteins.

\section{SDS-PAGE and western blotting}

Cells were grown in the growth conditions described in the Bacterial strains, plasmids, and growth conditions section until they reached the stationary phase, which took approximately 24 hours for E. coli and 48 hours for $P$. fluorescens. The cultures were separated into pellets and supernatants by double centrifugation from which the expression and secretion of the GFP-LARD fusion proteins were analyzed separately. Gel electrophoresis was undertaken according to Laemmli [40]. Fifteen micro-liters of cell extract or supernatant, equivalent to $15 \mu \mathrm{l} \mathrm{OD}_{600} \sim 2.5 \mathrm{cul}-$ ture broth $\left(0.0375 \mathrm{OD}_{600}\right.$ equivalent), was loaded on $10 \%(\mathrm{v} / \mathrm{v})$ SDS-PAGE, and western blotting was performed as described previously [11], using antiLARD or anti-GFP primary antibodies. The purified TliA $(99 \mu \mathrm{g} / \mathrm{ml})$ was diluted to 3.3 or $1.4 \mu \mathrm{g} / \mathrm{ml}$ and used as the internal standard for western blotting.

\section{Estimation of GFP fluorescence}

E. coli cells harboring pGFP-223, pGFP-LARD1 to 5, or pGFP-TliA was grown in $3 \mathrm{ml} \mathrm{LB}(50 \mu \mathrm{g} / \mathrm{ml}$ ampicillin $)$ at $37^{\circ} \mathrm{C}$ for $18 \mathrm{hr}$ or at $25^{\circ} \mathrm{C}$ for $36 \mathrm{hr}$. Different amounts of each culture were centrifuged, washed, and resuspended to meet $\mathrm{OD}_{600}=2$. One hundred microliters of the resuspended culture was added to Greiner 96 well Fluotrac ${ }^{\mathrm{TM}}$ for fluorescence measurement. Fluorescence was estimated with excitation at $485 \mathrm{~nm}$ and emission at 535 using a Tecan Genios Pro multifunction microplate reader. The relative fluorescence of each culture was obtained by comparing to that of the culture harboring pGFP-223. E. coli cells expressing different GFP fusion proteins were streaked on an LB plate, grown for 1 or 2 days, and photographed under long-wavelength UV light.

\section{Construction of the $P$. fluorescens knock-out mutant, $\Delta$ tliA} $P$. fluorescens SIK W1 $\Delta$ tliA was constructed using $s a c B$, a levansucrase-encoding gene from Bacillus subtilis, as a selection marker. Levansucrase catalyzes levan synthesis through transfructorylation of sucrose [41] and confers lethality to a variety of gram-negative bacteria [42]. The tliA gene was PCR amplified from pTOTAL and inserted into BamHI/HindIII site of pK18mobsacB, a vector with $s a c B$, $K m^{R}$, and a $m o b$ factor [43]. The plasmid was digested by $X h o I$ and SalI, which were in the middle of $t l i A$, and ligated to make an internal 512 bp-deficient mutant of tliA, $\Delta$ tliA. A constructed vector pKtliAXS with $\triangle$ tliA was transferred from $E$. coli S17-1 to P. fluorescens SIK W1 by conjugation using the $m o b$ factor. Since the replication origin of pK18mobsacB is inactive in P. fluorescens [43], the recombinant $P$. fluorescens was cultured with kanamycin and sought for the resistant strains with pKtliAXS inserted into the genome. That mutant strain conveyed sucrose sensitivity along with kanamycin resistance, implying a single crossover event between the intrinsic tliA and $\Delta t l i A$. In order to induce another crossover that replaced $t i A$ with $\triangle t l i A$ and eliminated $s a c B$ and $K m^{R}$ from the genome, a single colony of the mutant strain was grown in a nonselective LB medium and spread onto a $10 \%$ sucrose LB plate. $P$. fluorescens $\Delta$ tliA was selected as the colonies that grew only on the nonselective LB plate and had no lipase activity on a tributyrate plate.

\section{Abbreviations \\ TliA: Thermostable lipase A; ABC: ATP binding cassette; T1SS: Type 1 secretion system; LARD: Lipase $A B C$ transporter recognition domain; GFP: Green fluorescent protein; EGF: Epidermal growth factor; TliDEF: ABC transporter for TliA; PrtDEF: ABC transporter in Erwinia chrysanthemi; RTX: Repeat in toxins.}

\section{Competing interests}

The authors declare that they have no competing interests.

\section{Authors' contribution}

YP played leading role in writing the manuscript. YM played major role in design of experiments and western blot results. JR contributed to primer design, vector constructions and most experiments. NK and HC participated all experiments. JHA designed experiments and interpreted results. All authors read and approved the final manuscript. 


\section{Acknowledgments}

This work was supported by the Research \& Education Program funded by the Korean Ministry of Education, Science \& Technology. The authors would like to thank Kim JK for his help with laboratory techniques.

\section{Author details}

${ }^{1}$ Korea Science Academy of KAIST, 899 Tanggam 3-Dong, Busanjin-Gu, Busan 614-822, Korea. ${ }^{2}$ Department of Microbiology and Immunology, Medical Research Institute, Pusan National University School of Medicine, Yangsan 626-813, Korea.

Received: 24 January 2012 Accepted: 11 April 2012

Published: 11 May 2012

\section{References}

1. Delepelaire P: Type I secretion in gram-negative bacteria. Biochim Biophys Acta 2004, 1694(1-3):149-161.

2. Delepelaire P: PrtD, the integral membrane ATP-binding cassette component of the Erwinia chrysanthemi metalloprotease secretion system, exhibits a secretion signal-regulated ATPase activity. J Biol Chem 1994, 269(45):27952-27957.

3. Dinh T, Paulsen IT, Saier MH Jr: A family of extracytoplasmic proteins that allow transport of large molecules across the outer membranes of gramnegative bacteria. J Bacteriol 1994, 176(13):3825-3831.

4. Wandersman C, Delepelaire P: TolC, an Escherichia coli outer membrane protein required for hemolysin secretion. Proc Natl Acad Sci U S A 1990, 87(12):4776-4780.

5. Hung LW, Wang IX, Nikaido K, Liu PQ, Ames GF, Kim SH: Crystal structure of the ATP-binding subunit of an ABC transporter. Nature 1998, 396(6712):703-707.

6. Locher KP: Review. Structure and mechanism of ATP-binding cassette transporters. Philos Trans R Soc Lond B Biol Sci 2009, 364(1514):239-245.

7. Rees $D C$, Johnson $E$, Lewinson $O: A B C$ transporters: the power to change. Nat Rev Mol Cell Biol 2009, 10(3):218-227.

8. Binet $\mathrm{R}$, Wandersman $\mathrm{C}$ : Protein secretion by hybrid bacterial $A B C$-transporters: specific functions of the membrane ATPase and the membrane fusion protein. EMBO J 1995, 14(10):2298-2306.

9. Wandersman C: Secretion across the bacterial outer membrane. Trends Genet 1992, 8(9):317-322.

10. Ahn JH, Pan JG, Rhee JS: Identification of the tliDEF ABC transporter specific for lipase in Pseudomonas fluorescens SIK W1. J Bacteriol 1999, 181(6):1847-1852.

11. Chung CW, You J, Kim K, Moon Y, Kim H, Ahn JH: Export of recombinant proteins in Escherichia coli using $A B C$ transporter with an attached lipase ABC transporter recognition domain (LARD). Microb Cell Fact 2009, 8:11.

12. Felmlee T, Pellett $S$, Lee EY, Welch RA: Escherichia coli hemolysin is released extracellularly without cleavage of a signal peptide. J Bacteriol 1985, 163(1):88-93.

13. Su L, Chen S, Yi L, Woodard RW, Chen J, Wu J: Extracellular overexpression of recombinant Thermobifida fusca cutinase by alpha-hemolysin secretion system in E. coli BL21(DE3). Microb Cell Fact 2012, 11:8.

14. Palacios JL, Zaror I, Martinez P, Uribe F, Opazo P, Socias T, Gidekel M, Venegas A: Subset of hybrid eukaryotic proteins is exported by the type I secretion system of Erwinia chrysanthemi. J Bacteriol 2001, 183(4):1346-1358

15. Delepelaire $P$, Wandersman C: Protease secretion by Erwinia chrysanthemi. Proteases $B$ and $C$ are synthesized and secreted as zymogens without a signal peptide. J Biol Chem 1989, 264(15):9083-9089.

16. Duong F, Lazdunski A, Cami B, Murgier M: Sequence of a cluster of genes controlling synthesis and secretion of alkaline protease in Pseudomonas aeruginosa: relationships to other secretory pathways. Gene 1992, 121(1):47-54

17. Ghigo JM, Wandersman C: A carboxyl-terminal four-amino acid motif is required for secretion of the metalloprotease PrtG through the Erwinia chrysanthemi protease secretion pathway. J Biol Chem 1994, 269(12):8979-8985

18. Kuwahara K, Angkawidjaja C, Koga Y, Takano K, Kanaya S: Importance of an extreme C-terminal motif of a family 1.3 lipase for stability. Protein Eng Des Sel 2011, 24(5):411-418.

19. Omori K, Idei A, Akatsuka H: Serratia ATP-binding cassette protein exporter, Lip, recognizes a protein region upstream of the $C$ terminus for specific secretion. J Biol Chem 2001, 276(29):27111-27119.
20. Miyatake H, Hata Y, Fujii T, Hamada K, Morihara K, Katsube Y: Crystal structure of the unliganded alkaline protease from Pseudomonas aeruginosa IFO3080 and its conformational changes on ligand binding. J Biochem 1995, 118(3):474-479.

21. Baumann U, Wu S, Flaherty KM, McKay DB: Three-dimensional structure of the alkaline protease of Pseudomonas aeruginosa: a two-domain protein with a calcium binding parallel beta roll motif. EMBO J 1993, 12(9):3357-3364.

22. Baumann U: Crystal structure of the $50 \mathrm{kDa}$ metallo protease from Serratia marcescens. J Mol Biol 1994, 242(3):244-251.

23. Hamada K, Hata Y, Katsuya Y, Hiramatsu H, Fujiwara T, Katsube Y: Crystal structure of Serratia protease, a zinc-dependent proteinase from Serratia sp. E-15, containing a beta-sheet coil motif at $2.0 \mathrm{~A}$ resolution. J Biochem 1996, 119(5):844-851.

24. Fath MJ, Kolter R: ABC transporters: bacterial exporters. Microbiol Rev 1993, 57(4):995-1017.

25. Felmlee T, Welch RA: Alterations of amino acid repeats in the Escherichia coli hemolysin affect cytolytic activity and secretion. Proc Natl Acad Sci U S A 1988, 85(14):5269-5273.

26. Lilie H, Haehnel W, Rudolph R, Baumann U: Folding of a synthetic parallel beta-roll protein. FEBS Lett 2000, 470(2):173-177.

27. Ludwig A, Jarchau T, Benz R, Goebel W: The repeat domain of Escherichia coli haemolysin (HlyA) is responsible for its $\mathrm{Ca} 2+-$ dependent binding to erythrocytes. Mol Gen Genet 1988, 214(3):553-561.

28. Letoffe S, Wandersman C: Secretion of CyaA-PrtB and HlyA-PrtB fusion proteins in Escherichia coli: involvement of the glycine-rich repeat domain of Erwinia chrysanthemi protease B. J Bacterio/ 1992, 174(15):4920-4927.

29. Kwon HJ, Haruki M, Morikawa M, Omori K, Kanaya S: Role of repetitive nine-residue sequence motifs in secretion, enzymatic activity, and protein conformation of a family 1.3 lipase. J Biosci Bioeng 2002, 93(2):157-164

30. Guex N, Peitsch MC, Schwede T: Automated comparative protein structure modeling with SWISS-MODEL and Swiss-PdbViewer: a historical perspective. Electrophoresis 2009, 30(Suppl 1):S162-S173.

31. Meselson M, Yuan R: DNA restriction enzyme from E. coli. Nature 1968, 217(5134):1110-1114

32. Blenner MA, Shur O, Szilvay GR, Cropek DM, Banta S: Calcium-induced folding of a beta roll motif requires C-terminal entropic stabilization. J Mol Biol 2010, 400(2):244-256.

33. Letoffe $S$, Delepelaire $P$, Wandersman C: Protein secretion in gramnegative bacteria: assembly of the three components of $A B C$ protein-mediated exporters is ordered and promoted by substrate binding. EMBO J 1996, 15(21):5804-5811.

34. Chenal A, Guijarro Jl, Raynal B, Delepierre M, Ladant D: RTX calcium binding motifs are intrinsically disordered in the absence of calcium: implication for protein secretion. J Biol Chem 2009, 284 (3):1781-1789.

35. Debarbieux L, Wandersman C: Folded HasA inhibits its own secretion through its ABC exporter. EMBO J 2001, 20(17):4657-4663.

36. Bakkes PJ, Jenewein S, Smits SH, Holland IB, Schmitt L: The rate of folding dictates substrate secretion by the Escherichia coli hemolysin type 1 secretion system. J Biol Chem 2010, 285(52):40573-40580.

37. Choi HJ, Ahn JH, Park SH, Do KH, Kim J, Moon Y: Enhanced Wound Healing by Recombinant Escherichia coli Nissle 1917 via Human Epidermal Growth Factor Receptor in Human Intestinal Epithelial Cells: Therapeutic Implication using Recombinant Probiotics. Infect Immun 2011, 80(3):1079-1087.

38. Ahn JH, Pan JG, Rhee JS: Homologous expression of the lipase and $A B C$ transporter gene cluster, tliDEFA, enhances lipase secretion in Pseudomonas spp. Appl Environ Microbiol 2001, 67(12):5506-5511.

39. Kovach ME, Elzer PH, Hill DS, Robertson GT, Farris MA, Roop RM 2nd, Peterson KM: Four new derivatives of the broad-host-range cloning vector pBBR1MCS, carrying different antibiotic-resistance cassettes. Gene 1995, 166(1):175-176.

40. Laemmli UK: Cleavage of structural proteins during the assembly of the head of bacteriophage T4. Nature 1970, 227(5259):680-685.

41. Gay $P$, Le Coq D, Steinmetz M, Ferrari $E$, Hoch JA: Cloning structural gene $s a c B$, which codes for exoenzyme levansucrase of Bacillus subtilis: expression of the gene in Escherichia coli. J Bacteriol 1983, 153(3):1424-1431. 
42. Gay P, Le Coq D, Steinmetz M, Berkelman T, Kado Cl: Positive selection procedure for entrapment of insertion sequence elements in gram-negative bacteria. J Bacteriol 1985, 164(2):918-921.

43. Schafer A, Tauch A, Jager W, Kalinowski J, Thierbach G, Puhler A: Small mobilizable multi-purpose cloning vectors derived from the Escherichia coli plasmids pK18 and pK19: selection of defined deletions in the chromosome of Corynebacterium glutamicum. Gene 1994, 145(1):69-73.

doi:10.1186/1475-2859-11-60

Cite this article as: Park et al: Identification of the minimal region in

lipase ABC transporter recognition domain of Pseudomonas fluorescens

for secretion and fluorescence of green fluorescent protein. Microbial

Cell Factories 2012 11:60.

\section{Submit your next manuscript to BioMed Central and take full advantage of:}

- Convenient online submission

- Thorough peer review

- No space constraints or color figure charges

- Immediate publication on acceptance

- Inclusion in PubMed, CAS, Scopus and Google Scholar

- Research which is freely available for redistribution 